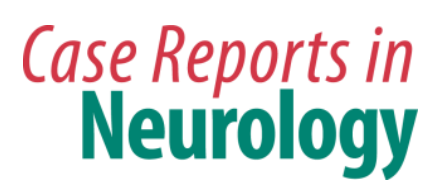

\title{
Vocalization in Dementia: A Case Report and Review of the Literature
}

\author{
Arkady Yusupov James E. Galvin \\ Alzheimer Disease Center, Departments of Neurology, Psychiatry and Population Health, \\ New York University Langone Medical Center, New York, N.Y., USA
}

\section{Key Words}

Alzheimer's disease - Dementia - Vocalization - Behavior and psychiatric symptoms of dementia $\cdot$ Nonpharmacologic interventions $\cdot$ Caregiver distress

\begin{abstract}
Background: Vocalizations are part of the spectrum of the 'negative' behavioral and psychological symptoms of dementia (BPSD). We describe a patient with moderate-stage mixed dementia of Alzheimer's disease and cerebrovascular disease and a left orbitofrontal lesion exhibiting vocalization. The use of 'redirection' has been demonstrated to be an effective nonpharmacological means of controlling BPSD, while reducing caregiver distress. Case Report: A 78-year-old right-handed African-American female presented with complaints of worsening memory and BPSD, causing significant caregiver distress. Throughout the evaluation, she constantly vocalized her son's name and made a continuous grunting noise, correlating with increased anxiety/agitation. We utilized a redirection technique, which achieved the immediate reduction of the vocalization symptoms. Caregiver psychoeducation was provided allowing them to use the redirection technique at home. Conclusions: In patients with dementia exhibiting negative symptoms of BPSD, using nonpharmacologic techniques (i.e. redirection) may be indicated. Psychotropic medications rarely address negative BPSD symptoms, while simultaneously decreasing patient's quality of life. Nonpharmacologic approaches are beneficial as first-line therapy for negative BPSD.
\end{abstract}

(C) 2014 S. Karger AG, Basel

\section{Introduction}

Vocalizations are part of the spectrum of 'negative' behavioral and psychological symptoms of dementia (BPSD) [1]. BPSD, also known as neuropsychiatric symptoms, constitute a 
major component of the dementia syndrome, regardless of the subtype. BPSD correlated strongly with the degree of functional and cognitive impairment and cause significant caregiver burden and distress [1]. It is estimated that BPSD affect up to $90 \%$ of all dementia subjects at some point during their illness, and is independently associated with poor outcomes, including distress among patients and caregivers, long-term hospitalization, misuse of medication, and increased healthcare costs [1]. As listed in table 1, BPSD can be divided into 'positive' symptoms such as agitation, aggression, hallucinations, and delusions and 'negative' symptoms such as passive resistance, apathy, wandering, and vocalizations.

Vocalizations consist of excessively loud and/or repetitive verbal utterances, such as single words or phrases, nonsensical sounds, screaming, moaning, and constant requests for attention [2-4]. Patients describe an inner urge or a local premonitory sensation, which increases anxiety or agitation. Anxiety and agitation are often relieved by performing the vocalization suggesting that these behaviors may provide a form of 'self-soothing'. Nearly all disruptive vocalizations are related to a form of brain injury; most have dementia due to Alzheimer's disease or cerebrovascular disease $[2,3]$. In susceptible persons, vocalizations can be triggered by a variety of stimuli, including the physical environment, stress, anxiety, or caregiver behaviors [5]. Thus, a vocalization could trigger a caregiver response that increases the patient's anxiety, leading to more vocalizations. The literature suggests a lack of efficacy for use of psychotropic medications for 'negative' symptoms of BPSD [1]. Due to the inability to direct oneself to the various stimuli that are affecting an altered central nervous system, use of psychotropic medications may not ease the symptoms of continuous vocalizations in patients with Alzheimer's disease and other forms of dementia. This report describes a case of vocalizations in moderate to severe Alzheimer's disease and use of 'redirection' as an effective nonpharmacologic means of controlling vocalizations and reducing caregiver distress.

\section{Case Presentation}

This case describes a patient with mixed Alzheimer's disease and cerebrovascular disease who developed the behavioral symptom of vocalization. The caregivers' primary concern at the initial office visit was the inability to control the patient's vocalizations with the use of psychotropic medications or direct orders to stop the behavior. The patient is a 78-year-old right-handed African-American female who presented for an evaluation of a memory and behavioral disorder. Significant past medical history includes hypertension, diabetes with neuropathy and severe retinopathy leading to visual impairment, and cerebrovascular disease. The patient completed a high-school education and was a retired licensed practicing nurse. The patient's daughter and son were the primary caregivers and served as the chief sources of information. The memory problems were first noted 9 months prior to the evaluation with increasing forgetfulness, anxiety, and agitation. One month after onset of cognitive symptoms, the patient was admitted to the hospital with right hemiparesis. A carotid endarterectomy was performed and the patient was transferred to subacute rehabilitation. During her stay at the facility, she developed increased agitation and started vocalizing stereotyped repetition of phrases which have persisted. At the initial visit, the family reported that the patient was not oriented to time or place, demonstrated poor problem-solving abilities, social withdrawal, limited household functioning, and was doubly incontinent. Behavioral changes included verbal aggression, fearfulness, anxiety, and vocalizations, repeating the same word over again (typically her son's name) with increased volume and agitation. There were past episodes of visual hallucinations reported at the 
Yusupov and Galvin: Vocalization in Dementia: A Case Report and Review of the Literature

subacute facility treated with haloperidol and quetiepine that had no effect on the vocalizations. The behavioral features led to moderately severe caregiver burden and stress. At the time of evaluation, she was treated with donepezil, memantine, venlafaxine, and intermittent use of lorazepam.

Neuroimaging studies were performed during and after the hospitalization. On admission, an MRI scan demonstrated cortical and hippocampal atrophy (fig. 1a), a lacunar infarct in the left basal ganglia and internal capsule (fig. 1b), and ischemic white matter changes. An acute left occipital infarct was detected (fig. 2). More importantly, a subacute infarct was detected in the left rectus gyrus/medial orbital frontal gyrus (arrows in fig. 2).

On examination, the patient was physically and visually impaired and confined to a wheelchair. Her pupils were nonreactive. Facial movement and sensation were intact and her tongue was midline without fasciculations. On motor exam, she had normal muscle bulk and tone, mild bradykinesia and full strength. She had mild dysmetria and dysdiadochonesia. Deep tendon reflexes were traced throughout and no pathologic reflexes were noted. Limited neurocognitive testing was performed due to visual impairment. She was not oriented to date or place of examination. She could not spell 'WORLD' backwards or perform serial subtraction. She scored 4 (out of a possible 22) on the Visually Impaired Version of the Montreal Cognitive Assessment. She recalled 11 of 36 items on the Hopkins Verbal Learning Task, immediate recall but 0 of 12 items on the delayed recall.

During examination, the patient would constantly vocalize her son's name, progressing from normal conversational tone to shouting. She would also make distinct grunting noises, which seemed to correspond to increasing anxiety levels. A redirection and relaxation technique was attempted to get the patient to discontinue her vocalizations. The patient was called to attention by calling her first name, and then was directed to take a deep breath and count to 10 . This technique was able to break the vocalization cycle for 10- to 15-min intervals. Each time the patient was redirected, the resulting vocalization-free interval was longer and achieved faster. During these periods, the patient was more lucid and able to answer questions and follow directions. These techniques were demonstrated to the caregivers, who successfully performed the redirection techniques.

At the 3-month follow-up, the caregivers reported continued relief of vocalization symptoms with constant use of the redirection technique. The patient's anxiety levels and overall psychological well-being were drastically improved with the ability to control her vocalization symptoms. Caregiver distress was also reduced as vocalizations diminished in frequency and intensity. Six months later, vocalizations persisted, but redirection techniques still offered benefit by reducing the length and volume of each vocalization behavior. By 12 months, low doses of risperidone were required to reduce agitation, which often triggered vocalization episodes.

\section{Discussion}

Neuroanatomical correlates of vocalizations may involve damage or injury to (1) orbitofrontal structures leading to disinhibition and (2) dorsolateral prefrontal structures leading to deficits in decision-making. This patient had a subacute infarct in the left rectus gyrus/medial orbitofrontal gyrus region (Brodmann areas 11-12). The orbitofrontal gyrus is associated with sensory integration, affect, decision-making and hedonism. Lesions in this region can lead to cognitive, behavioral and emotional symptoms [5]. It is likely that the new onset of anxiety and vocalization behavior is due to this subacute infarct. 
We found that the redirection and relaxation technique immediately worked with the patient during the initial office visit. The patient was able to focus in on her breathing, decreased her anxiety, and allowed for the repetitively loud name calling and grunting to subside for 10- to 15-min intervals. Successive attempts at redirection were used as the patient's vocalization symptoms reappeared. Each redirection attempt resulted in a longer vocalization-free period, in which the patient expressed relief in her ability to stop vocalizing. The caregivers were educated on the use of redirection and encouraged to use this method in the home environment.

This patient had several risk factors for developing vocalizations: moderate-stage Alzheimer's disease, extensive cerebrovascular disease, and physical limitations (wheelchairbound due to neuropathy, and visual impairment due to retinopathy). Medications are often of limited value in the management of disruptive vocalizations and other negative BPSD symptoms. Benzodiazepines should be prescribed with great caution; there are no objective data of effectiveness, and side effects are common. Low serotonin levels have been associated with lack of impulsive control, thus providing a rationale for use of medications with serotonergic properties (e.g., buspirone, trazodone, and selective serotonin reuptake inhibitors) [6]. Possible pharmacological interventions are listed in table 1. However, medications should be used only as an adjunct to behavioral management techniques to control negative BPSD.

In a multicenter, observational study of five Italian nursing homes, randomized selections of 87 daily nursing shifts were evaluated. Nurses were asked to keep daily diaries and record duration of vocalization episodes encountered, and management strategies. During the total observation time (36,540 $\mathrm{min}), 23.6 \%$ (8,653 $\mathrm{min}$ ) of nursing care time involved working with and managing disruptive vocalization patients. The nurses recorded an average of 6.5 (302/46) vocalizations on morning shifts and 7.3 (302/41) during afternoon shifts, with an average duration of about a quarter of an hour each. Managing disruptive vocalizations with multiple strategies reduced the duration of the vocalization episode and increased the perceived effectiveness of management [7].

Current guidelines recommend nonpharmacological interventions as first-line treatment for BPSD, followed by the least harmful medication for the shortest time possible [8]. Nonpharmacological interventions (table 1) in the management of BPSB have been classified into the following categories (i) cognitive-/emotion-oriented interventions (reminiscence therapy, simulated presence therapy, validation therapy); (ii) sensory stimulation interventions (acupuncture, aromatherapy, light therapy, massage/touch, music therapy, transcutaneous electrical nerve stimulation); (iii) behavior management (distraction, redirection, relaxation) techniques, and (iv) other psychosocial interventions such as animal-assisted therapy and exercise (table 1). Benefits from psychoeducational interventions for caregivers were documented to be long lasting, especially when delivered individually. A study of residents in residential aged care facilities found that there is growing evidence on the risks associated with psychotropics, including falls, pneumonia, hospitalization and mortality [9].

\section{Conclusion}

Use of psychotropic medications has become common practice in the treatment of neuropsychiatric symptoms such as vocalizations. Inhibiting a patient through the use of sedatives will undoubtedly prevent a patient from exhibiting vocalizations. However, this is not a solution to the problem due to serious negative implications to quality of life and potentially adverse side effects. Based on the results of this case report, using the nonphar- 
macologic approach of redirection in an attempt to break the vocalization pattern is recommended as a first-line intervention and may delay or reduce the need for antipsychotic medications.

\section{Acknowledgements}

This project was supported by grants from the National Institutes of Health - P30 AG008051 and R01 AG040211 and a grant from the New York State Department of Health (DOH-2011-1004010353).

\section{Disclosure Statement}

None of the authors have personal, financial or potential conflicts of interest.

\section{References}

1 Cerejeira J, Lagarto L, Mukaetova-Ladinska EB: Behavioral and psychological symptoms of dementia. Front Neurol 2012;3:73.

-2 Cariaga J, Burgio LD, Flynn W, Martin D: A controlled study of disruptive vocalization among geriatric residents in nursing homes. J Am Geriatr Soc 1991;39:501-507.

3 Cohen-Mansfield J, Werner P, Marx M: Screaming in nursing home residents. J Am Geriatr Soc 1990;38:785792.

4 Ryan DP, Tainsh SMM, Kolodny V, Lendrum BL, Fisher RH: Noise-making amongst the elderly in long term care. Gerontologist 1988;28:369-371.

5 Kringelbach ML: The orbitofrontal cortex: linking reward to hedonic experience. Nat Rev Neurosci 2005;6:691-702.

-6 Sloane P, Davidson S, Buckwalter K, Linsey A, Ayers S, Lenker V, et al: Management of the patient with disruptive vocalization. Gerontologist 1997;37:675-682.

7 Palese A, Menegazzo E, Baulino F, Pistrino R, Papparotto C: The effectiveness of multistrategies on disruptive vocalization of people with dementia in institutions: a multicentered observational study. J Neurosci Nurs 2009;41:191-200.

-8 Gauthier S, Cummings J, Ballard C, Brodaty H, Grossberg G, Robert P, et al: Management of behavioral problems in Alzheimer's disease. Int Psychogeriatr 2010;22:346-372.

-9 Hilmer S, Gnjidic D: Rethinking pyschotropics in nursing homes. Med J Aust 2013;2:77. 
Yusupov and Galvin: Vocalization in Dementia: A Case Report and Review of the Literature

Table 1. BPSD and approaches to management

\begin{tabular}{ll}
\hline $\begin{array}{l}\text { Negative symptoms } \\
\text { Vocalizations, screaming }\end{array}$ & $\begin{array}{l}\text { Positive symptoms } \\
\text { Agitation, aggression }\end{array}$ \\
$\begin{array}{l}\text { Passive resistance } \\
\text { Wandering }\end{array}$ & $\begin{array}{l}\text { Hallucinations } \\
\text { Delusions } \\
\text { Purposeless hyperactivity }\end{array}$ \\
$\begin{array}{l}\text { Apathy } \\
\text { Social isolation, withdrawal }\end{array}$ & $\begin{array}{l}\text { Disinhibition } \\
\text { Depression, anxiety }\end{array}$ \\
\hline $\begin{array}{l}\text { Nonpharmacologic interventions } \\
\text { Behavioral management techniques (distraction, redirection, } \\
\text { relaxation) }\end{array}$ & Pharmacologic interventions \\
$\begin{array}{l}\text { Cognitive/emotion-oriented approaches (reminiscence therapy, } \\
\text { validation therapy, stimulated presence therapy) }\end{array}$ & Antipsychotic (classic and atypical) \\
$\begin{array}{l}\text { Sensory stimulation interventions (acupuncture, aromatherapy, } \\
\text { light therapy, massage/touch therapy, music therapy) }\end{array}$ & Cholinesterase inhibitors \\
$\begin{array}{l}\text { Psychoeducational approaches for caregivers } \\
\text { Montessori-based activities (memory BINGo, group sorting) }\end{array}$ & Memantine \\
\begin{tabular}{l} 
Adult day programs \\
\hline
\end{tabular} & $\begin{array}{l}\text { Antidepressants } \\
\text { Anticonvulsants }\end{array}$ \\
\hline
\end{tabular}
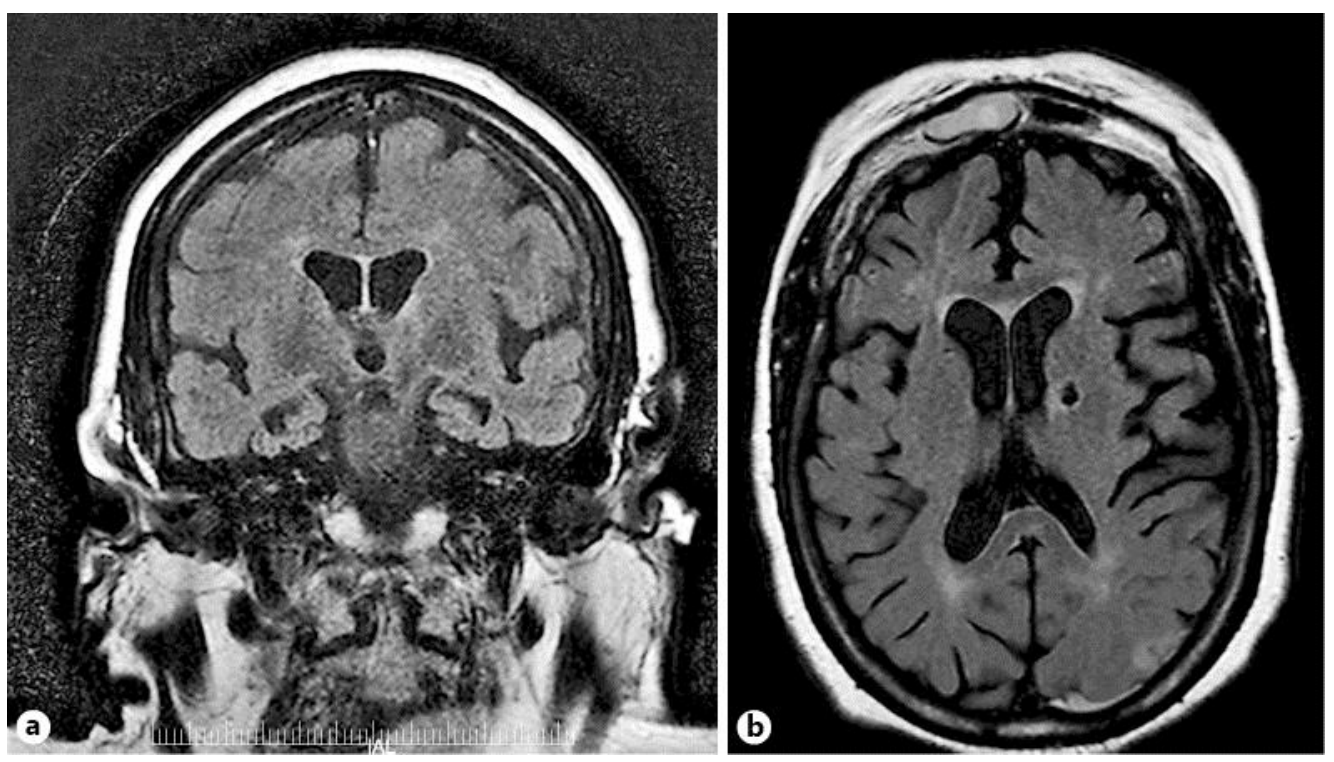

Fig. 1. Admission MRI supporting the diagnosis of mixed dementia of Alzheimer's disease and cerebrovascular disease. a Coronal FLAIR image demonstrating mild cortical and hippocampal atrophy. b Axial FLAIR image demonstrating a chronic lacunar infarct involving the left basal ganglia and posterior limb of the internal capsule and periventricular ischemic white matter changes. 
Case Rep Neurol 2014;6:126-133

DOI: $10.1159 / 000362159$


Yusupov and Galvin: Vocalization in Dementia: A Case Report and Review of the
Literature
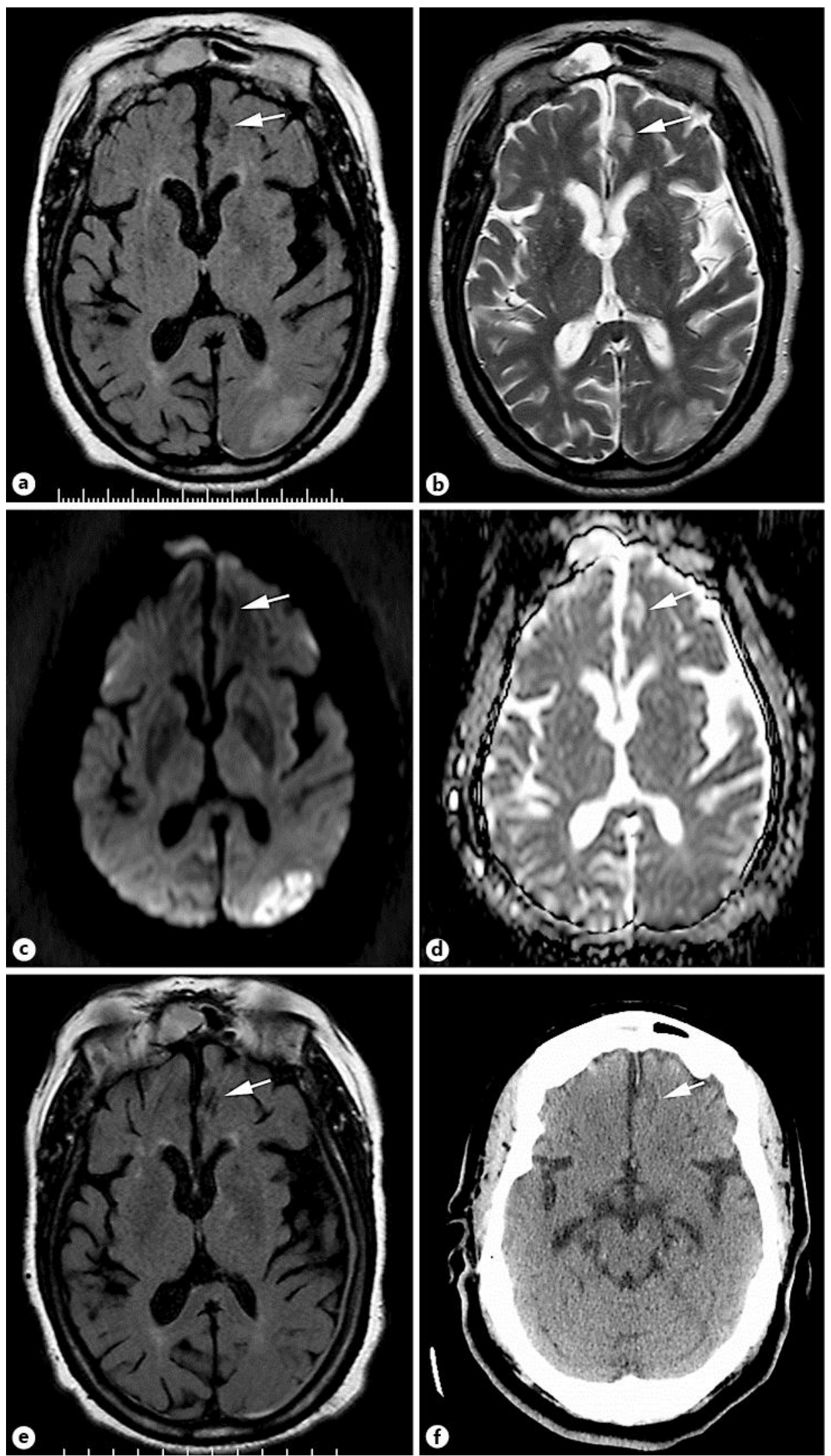

Fig. 2. Orbitofrontal infarct possibly linked to vocalization behaviors. On admission to the hospital, an

KARGER 
Yusupov and Galvin: Vocalization in Dementia: A Case Report and Review of the Literature

acute left occipital infarct was noted. Incidentally, a subacute infarct was detected in the left rectus gyrus/medial orbital frontal gyrus (arrows). a Axial FLAIR. b Axial DWI. c Axial T2 TSE. d Axial dADC. e Axial FLAIR image on follow-up MRI, 2 months after hospitalization. $f$ Axial noncontrast CT scan performed 3 months after hospitalization. 